\title{
The mass distribution in the innermost regions of Spiral Galaxies.
}

\author{
Charu Ratnam \& Paolo Salucci \\ International School for Advanced Studies, SISSA, Via Beirut 2-4, I-34013 \\ Trieste, Italy
}

\begin{abstract}
We use high-spatial resolution $(\sim 100 p c)$ rotation curves of 83 spiral galaxies to investigate the mass distribution of their innermost kpc. We show that, in this region, the luminous matter completely accounts for the gravitational potential and no dark component is required. The derived I-band disk mass-to-light ratios $\mathcal{Y}_{I}$ agree well with those obtained from population synthesis models and correlate with color in a similar way. We find strict upper limits of $\sim 10^{7} M_{\odot}$ for the masses of compact bodies at the center of spirals, ruling out that these systems host the remnants of the quasar activity.
\end{abstract}

\section{Introduction}

There is increasing direct evidence that, at the centers of bulge-dominated galaxies there reside Massive Dark Objects (MDO), which are probably the remnants of the engines that once powered the QSO phenomenon (Ho, 1998; Kormendy \& Richstone, 1995). In fact, virtually every hot galaxy hosts a $\mathrm{MDO} / \mathrm{BH}$ with a mass ranging from $\sim 10^{8} M_{\odot}$ to $2 \times 10^{10} M_{\odot}$, similar to those related to the QSO phenomenon. For disk galaxies, the situation is different and much more uncertain. A direct determination of the central mass has been obtained only in very few cases which include our own Galaxy where a black hole with a mass $2 \times 10^{6} M_{\odot}$ has been discovered (Ghez et al.1999, Genzel 1998; see also Salucci et al 1999 for other few cases). Remarkably, these masses do not exceed $10^{7} M_{\odot}$; however, the lack of detections of very massive objects $\left(M_{M D O}>10^{8} M_{\odot}\right)$ cannot be ascribed to observational biases. In fact, the rotation curves $(\mathrm{RC})$, in great number available down to $r_{i n} \sim 100 p c$, could easily expose central bodies with masses of the order of $\sim 10^{8} M_{\odot}$, given that usually $V\left(r_{i n}\right) \sim 10 \mathrm{~km} / \mathrm{s}$, or equivalently, the stellar mass inside $100 \mathrm{pc}$ barely reaches $10^{7} M_{\odot}$. On the other hand, the same $\mathrm{RC}$ analysis determining the MDO mass, obtain also the mass distribution of the innermost regions. This 
is particularly important in the case of low luminosity DM-dominated spirals (see Persic and Salucci, 1990) for which it is generally difficult to disentangle the disk component from the whole bulk of gravitating matter. In any cases, let us stress that to proper model the region where the luminous matter dominates is indispensable to infer the structural properties of dark matter.

The aim of this work is to study the innermost kinematics of spirals in order to a) investigate the distribution of matter in relation to that of light $b$ ) set stringent upper limits on the MDO masses. The plan of the paper is the following: in section 2 we analyse a sample drawn from the 967 rotation curves (RC's) of Persic and Salucci (1995), in section 3 we derive and discuss the disk properties and in sections 4 and 5 we derive upper limits on the MDO/BH masses and discuss the results. In this paper we assume $H_{0}=75 \mathrm{~km} / \mathrm{s} \mathrm{Mpc}^{-1}$, and take, as the reference magnitude in the B-band, $M_{*}=-20.5$, which translates to $M_{*}-21.9$ in the I-band (e.g. Rhee, 1997). All luminosities correspond to the I-band unless otherwise specified.

\section{Inner Rotation Curves of Late Types Spirals and mass modeling}

Recently, about a thousand rotation curves of spiral galaxies (PS95), tracing the kinematics inside the central kpc, have been available. Among these, about one hundred have at least one measurement $<200 p c$ and several $<500-$ 1000 pc. For these objects the detection of a MDO of $\sim 10^{8} M_{\odot}$ or more, if present, is guaranteed and so is the determination of the stellar disk mass, provided that in this region the stellar disk is the major mass component.

Therefore, we select from PS95 the largest sample of rotation curves equally distributed in magnitude interval and of sufficient high-quality for our purposes, by setting the following criteria: $i$ ) each $\mathrm{RC}$ has at least 4 measurements inside a radius of $340\left(1-\frac{\left(M_{I}+15\right)}{9}\right) p c \sim(350-500) p c$, ii) for each $\mathrm{RC}$, the innermost data is situated at a radius $r_{i n}<100\left(1-\frac{\left(M_{I}+15\right)}{18}\right) \sim(100-150) p c$. Let us notice that to restrict the criteria reduces the number of RC's without improving their already high quality that however rapidly decreases as they are relaxed. The rotation curves resulting from the selection are generally smooth, axisymmetric and with negligible non circular perturbations. It is evident that there is a very good agreement between the rotation fields on the receding and the approaching arm (see PS95). The sample (Sample B in Salucci et al., 2000) has 83 objects, well mixed in luminosity and almost equally distributed between Sb-Sc and Sd-Im Hubble types.

We aim to reproduce the RCs by a mass model featuring: (i) a Freeman disk of length-scale $R_{D}$, (derived in PS95) and mass $M_{D}$, which contributes to the 

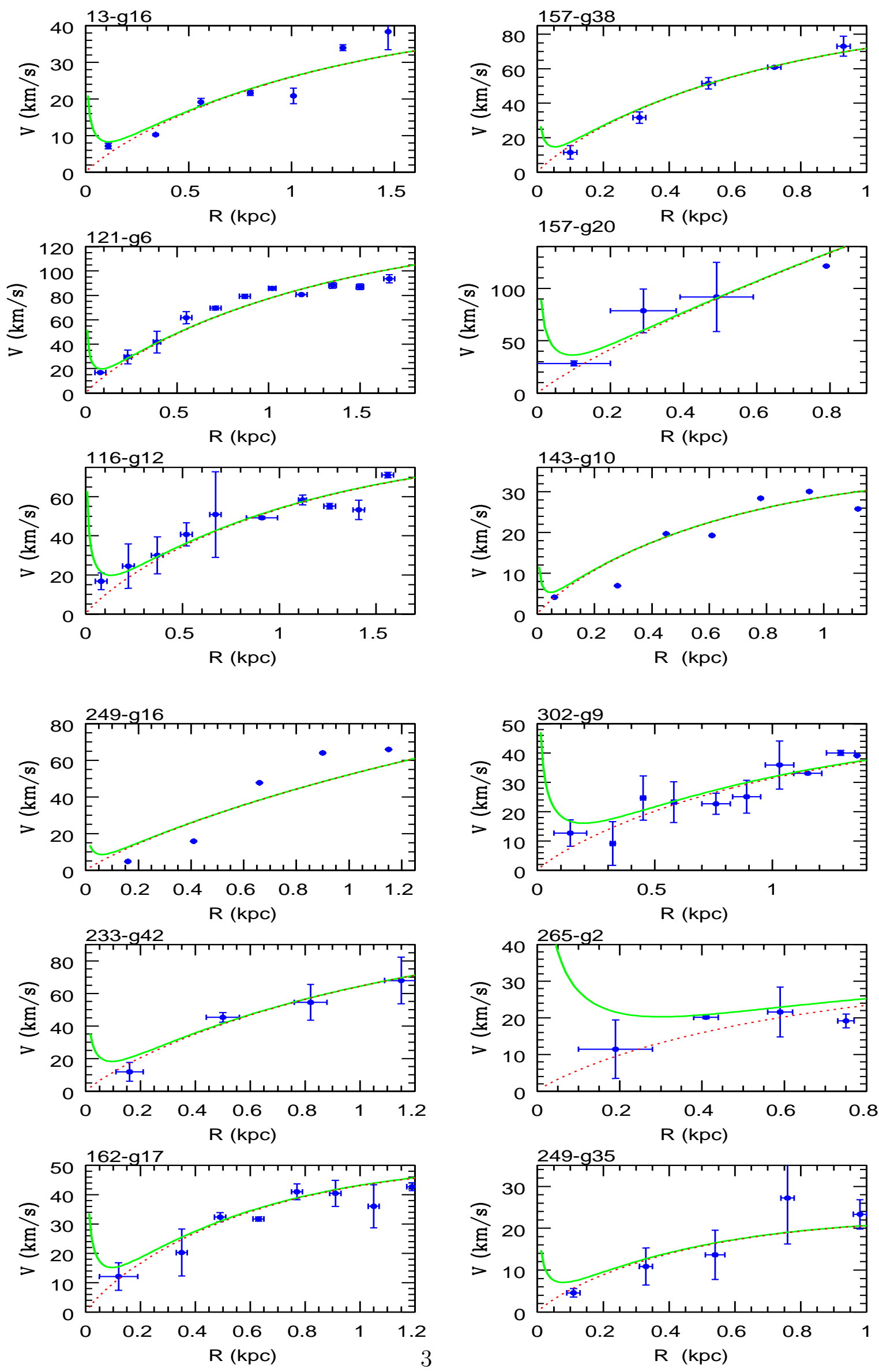

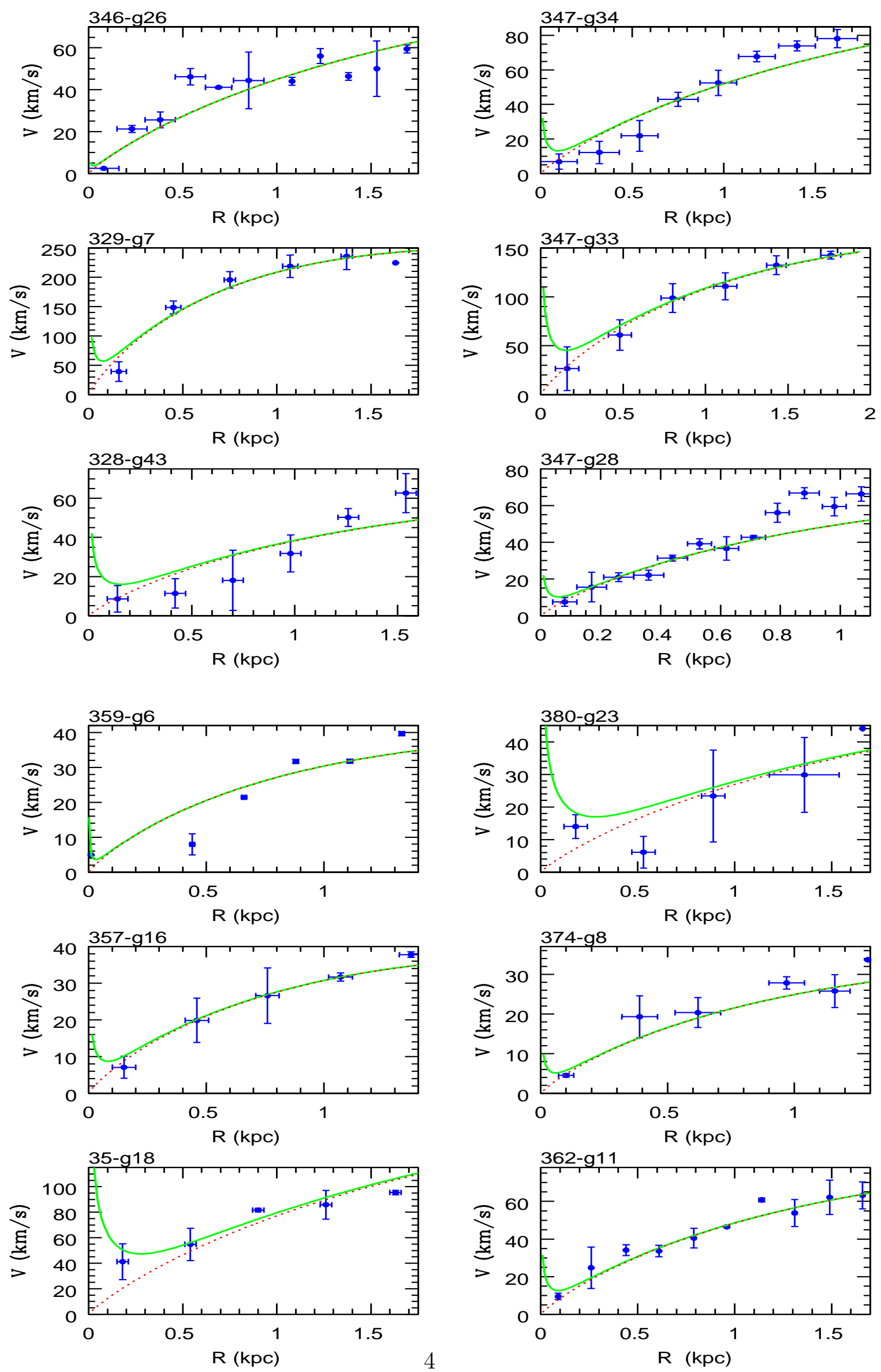

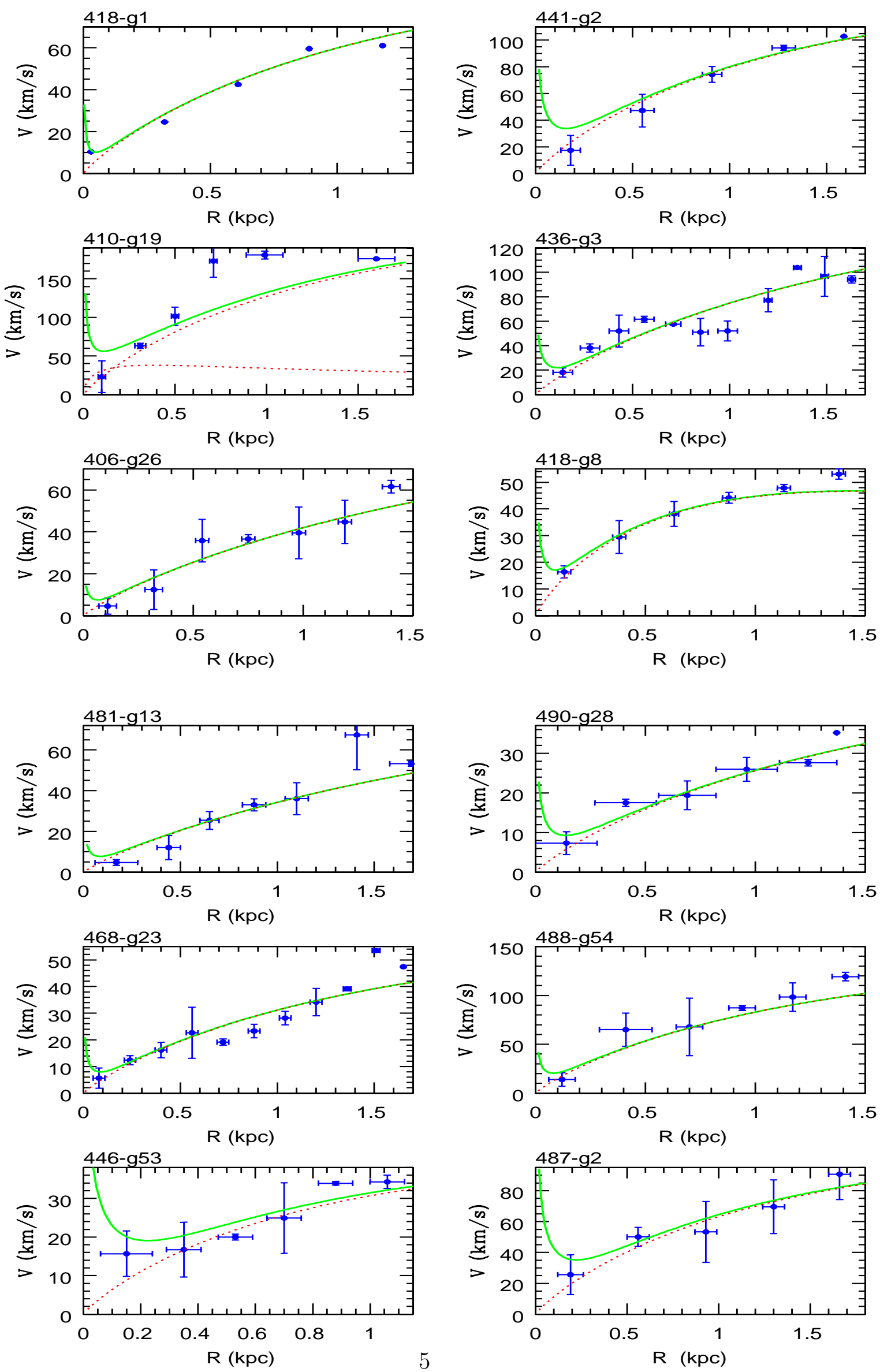

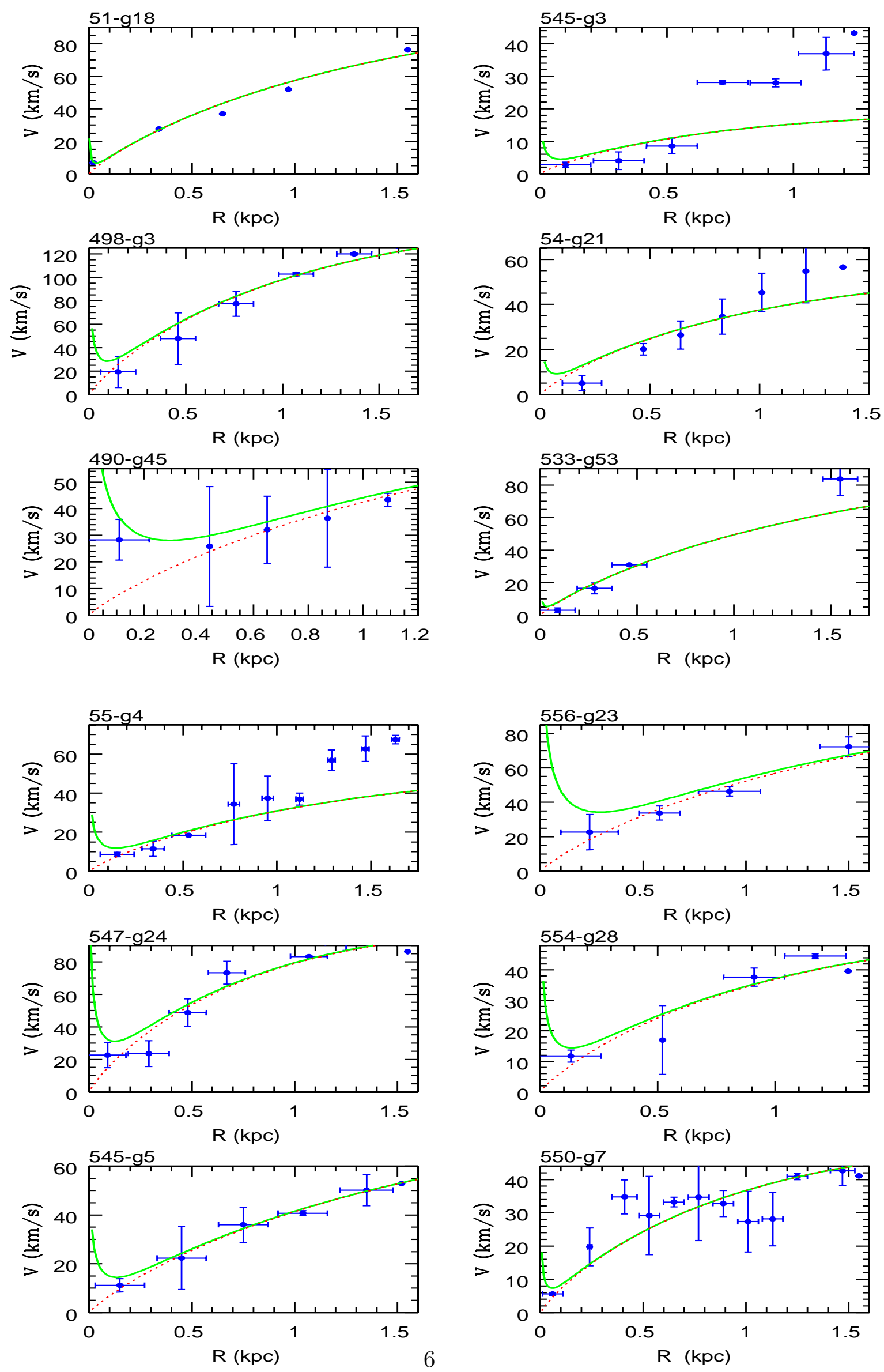

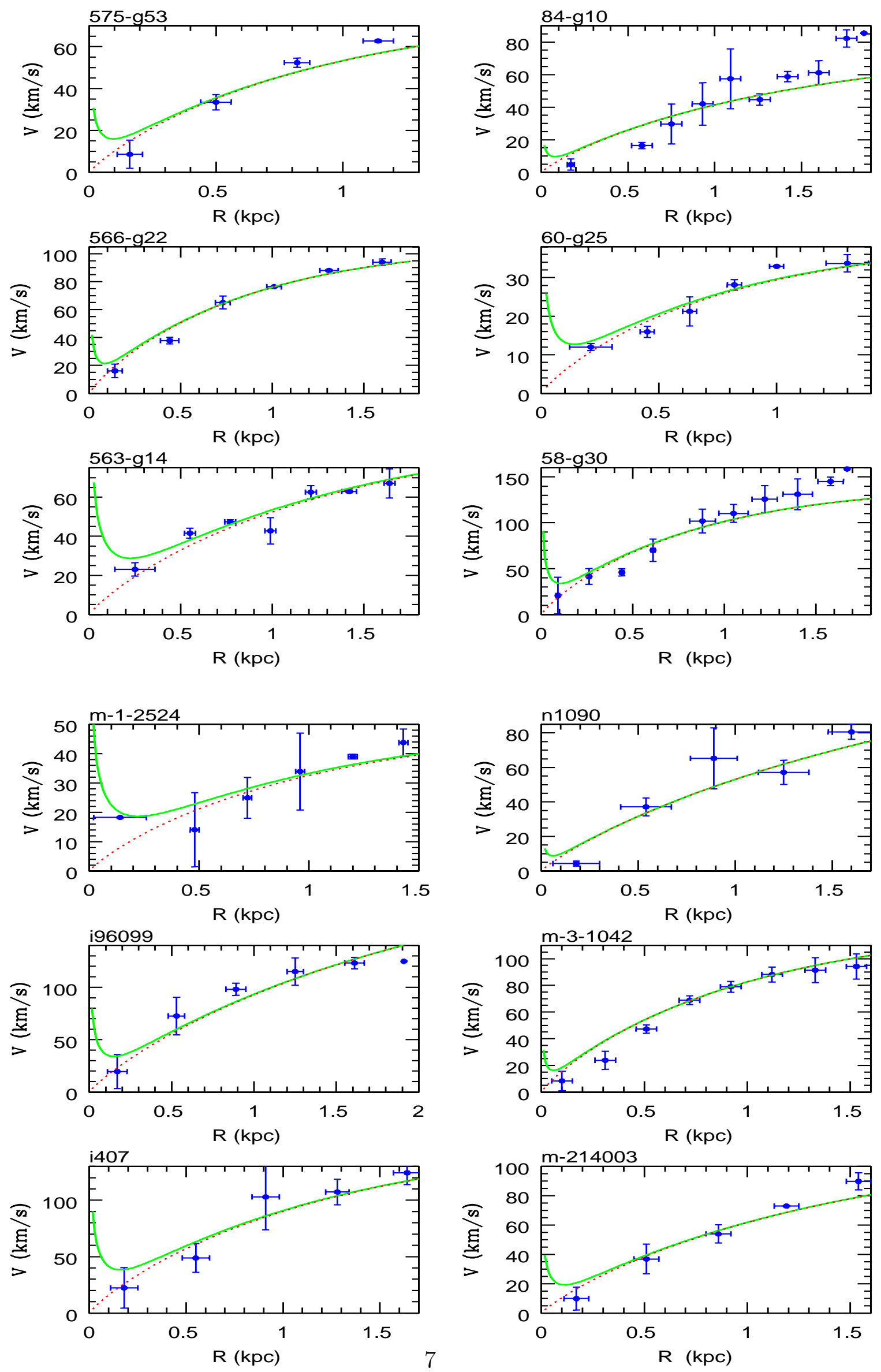

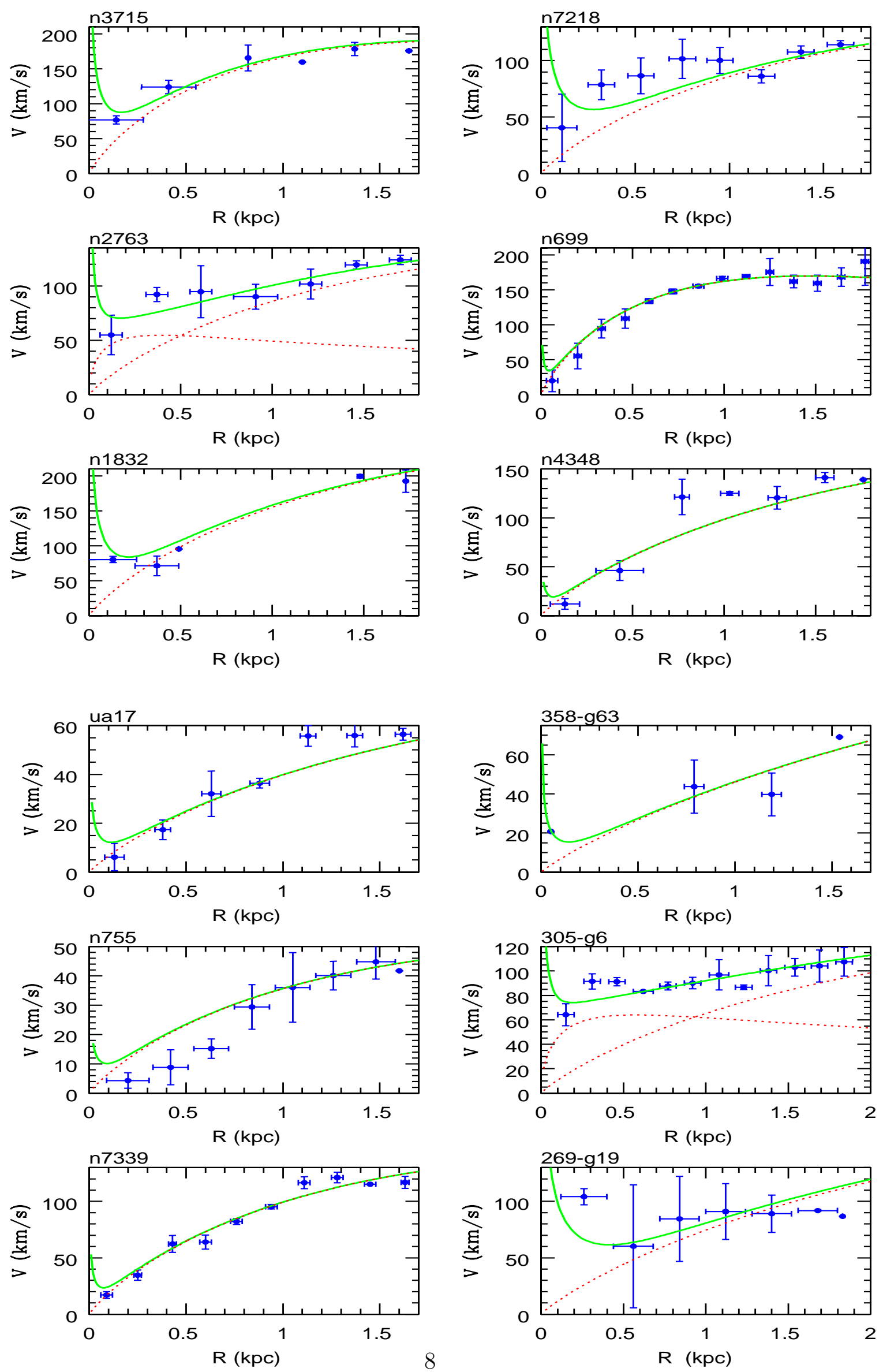

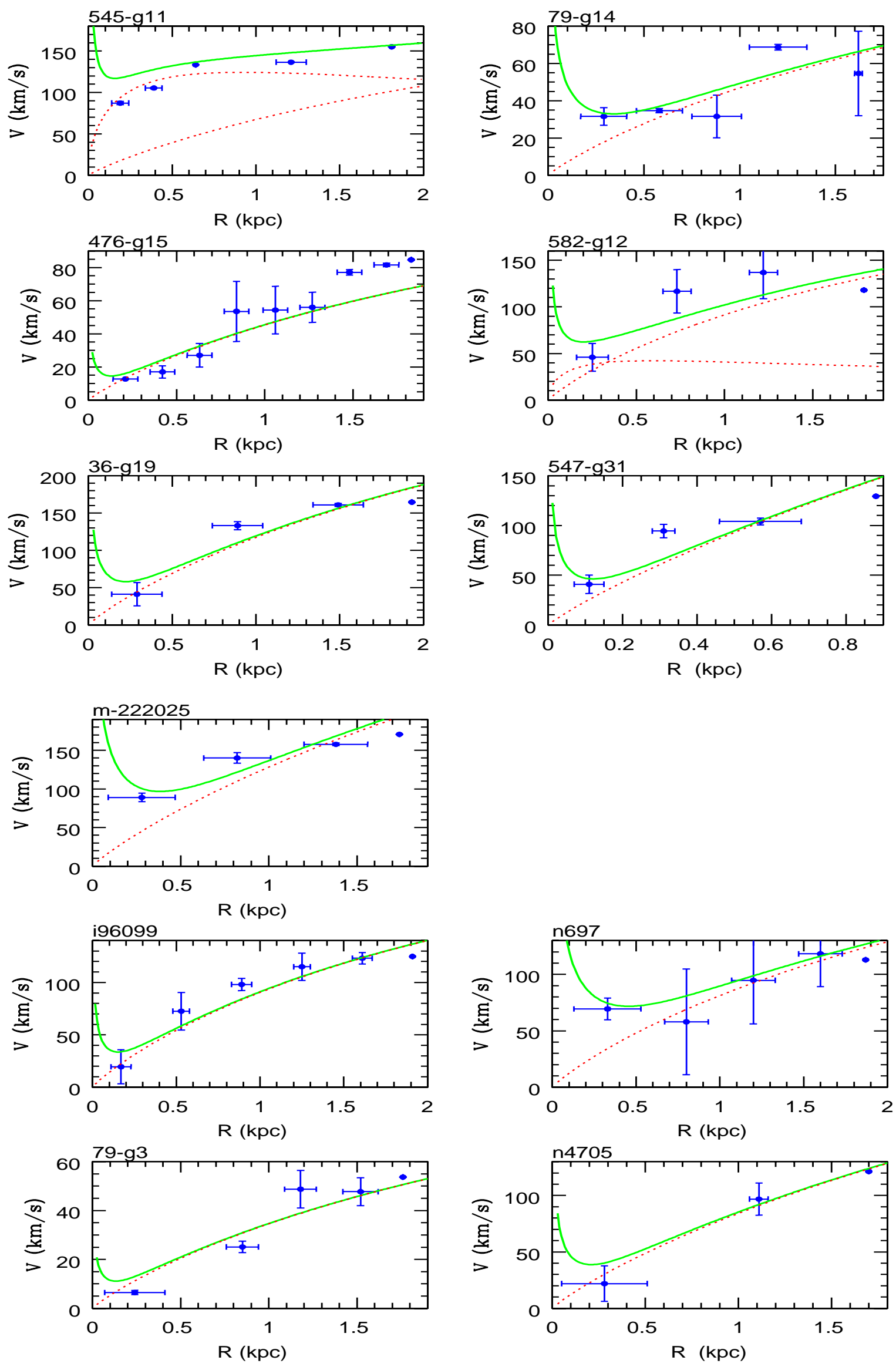

Fig. 1. Mass models of the 83 objects of sample B. The dashed lines indicate the OD model (in a few cases a bulge has been included) while the solid line indicates the disk $+\mathrm{BH}$ model. 
circular velocity as:

$$
V_{d}^{2}(R)=\frac{1}{2} G M_{D} / R_{D} x^{2}\left(I_{0} K_{0}-I_{1} K_{1}\right)_{x / 2}
$$

where $I_{n}, K_{n}$ are modified Bessel functions and $x=R / R_{D}$, and (ii) a MDO which contributes to $V(R)$ as:

$$
V_{M D O}^{2}(R)=G M_{M D O} / R
$$

where the MDO mass is:

$$
M_{M D O}=f G^{-1} V^{2}\left(r_{i n}\right) r_{i n}
$$

with $0<f \leq 1$.

In Fig.(1) we compare the velocity data with the best-fitting mass models in the cases of 1) $f=0$ (only disk, OD) mass model and of 2) $f>0$ (disk+ black hole) mass model (in practice: $f \simeq 1$ ). As result, we find no centralbody-dominated (CBD) rotation curve, i.e. no RC shows the Keplerian fall-off expected for a CBD RC. On the contrary, rotation curves are strikingly close to those predicted by the OD model, i.e. by a self-gravitating exponential thin disk with radially constant mass-to-light ratio. The results of the mass modeling are: in 57 objects the OD velocity curve accounts for the rotation data in a excellent way and it lies within the data error bars (for $R<R_{I B D}$ see below). In 14 cases ${ }^{\top}$ the luminous matter accounts for the rotation curve in a satisfactory way: data and model differ utmost by $2 \sigma$ and no evidence for an additional component emerges. In 9 cases $^{2}$ the OD fits are reasonable expecially if we consider that the corresponding RC's have non-negligible internal dispersion and/or some asymmetry. The low-luminosity galaxy 545-G3 is dominated by dark matter at any radius. Finally, in 2 cases; 346-G26, 410-G19, the OD model reproduces the data with some difficulty.

A simple inspection of the results shows that in every galaxy only for $R \geq$ $R_{I B D}{ }^{3}$ the dark component begins to significantly contribute to the total gravitational potential. We then confirm the picture of Salucci \& Persic (1999) and Salucci etal, (2000) according to which, the luminous matter dominates an innermost region of spirals of size $R_{I B D}$. This truly baryonic scale turns out to be a function of galaxy luminosity by ranging from $0.5 R_{D} \sim 0.5 k p c$ to $2 R_{D} \sim 30 k p c$ along the luminosity sequence Such a transition can be seen, e.g. in the RC of 55-G4: OD curve reproduces the data only out to $R=R_{I B D} \sim 0.5 k p c$. More in general, the best-fit models (see Fig 1) clearly

1 349-G6, 38-G23, 35-G18, 157-G20, 436-G3，468-G23，547-G24，550-G7，N4348, 358G-63, 582-G12, 547-G31, I96099, N4705

2 347-G84, 328-G43, 249-G16, 84-G10, N755, 79-G14, M222025, N7218, 249-G16

3 Inner Baryon Dominance 


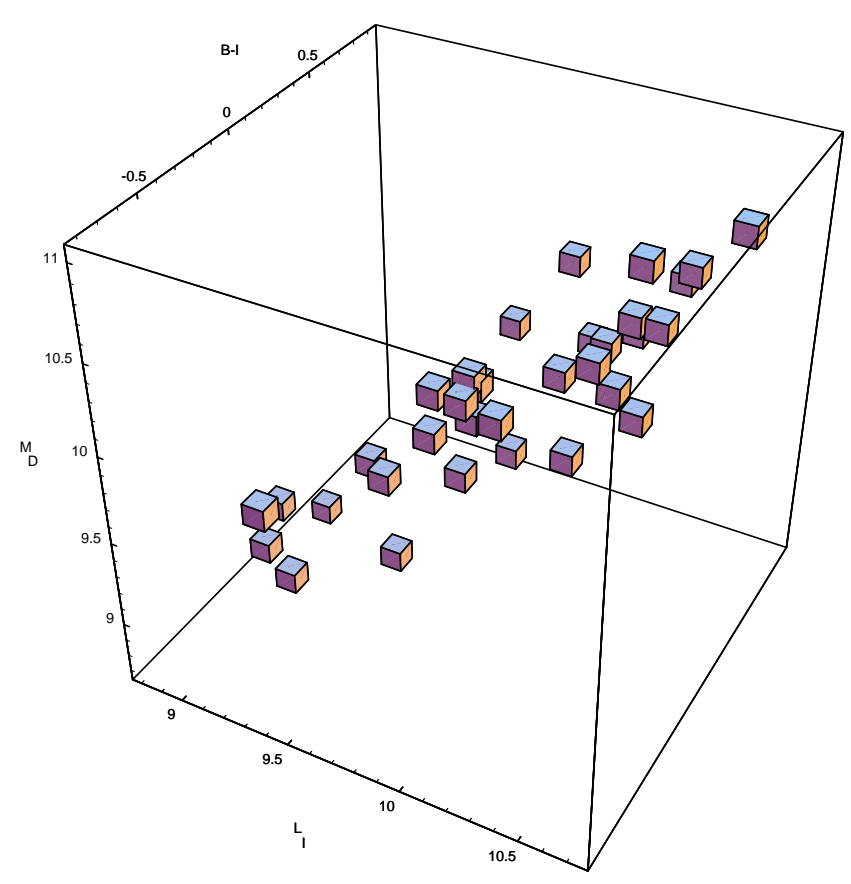

Fig. 2. The 3D space of galaxy properties. $M_{D}-(B-I)-L_{I}$.

show that, with the exception of the least luminous galaxy, at $R \sim 0.5 \mathrm{kpc}$, the DM contribution to the RC lies below the detection threshold. On the other hand, in order to become the major component at a $2-3$ disk scale-lenghts, the dark mass, negligible at $0.5 k p c$ must strongly increase with radius.

We conclude by stating that the OD mass models reproduce the innermost kpc of the rotation curves extremely well: on this scale, there is it no hint of a dark component.

\section{Disk properties}

Disk masses are derived from eq (1); Although the uncertainties on the disk mass-to-light ratio values must include model-fit uncertainties, photometry errors, and uncertainties on the assumed inclination and distance (the latter present also for Tully-Fisher distances). The uncertainty budget can be roughly estimated as: $\delta M_{D} / L_{I} \simeq 0.4 M_{D} / L_{I}$.

The present sample of DM-free mass models is a most suitable one for investigating the disk masses in relation with the photometric properties of galaxies. In detail, this is done with a sub-sample of Sample B comprising 28 objects for which $I$-band luminosities and $B-I$ colors are available. In Table 1 we report best-fit disk masses, color, mass-to-light ratios $M_{D} / L_{I}$ and luminosity for these objects. B-I color are good indicators of stellar mass-to-light ratio 


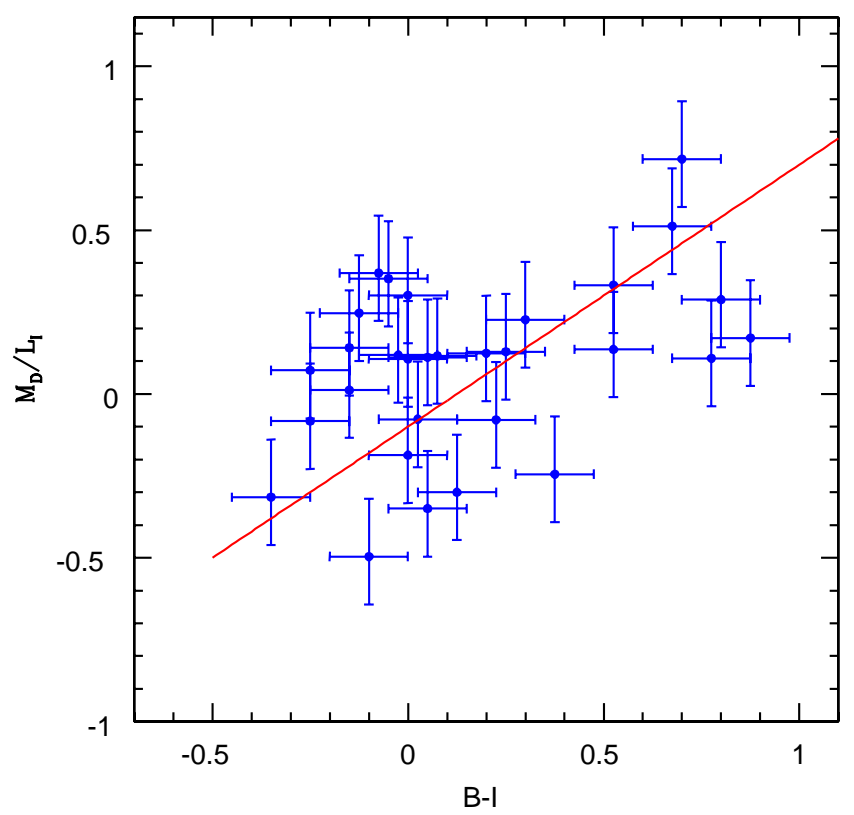

Fig. 3. Logarithm of the mass-to-light ratio in the I band as a function of the B-I color. The solid line is from Vazdekis etal. 1996. The uncertainty in B-I is 0.1 mag (Vazdekis et al 1996). In Fig.2 we the show $\mathcal{Y}_{I}$ vs. $B-I$ colors alongside with with the same relation predicted by population synthesis models. We find

$$
\mathcal{Y}_{I} \simeq 0.63 \times\left(\frac{1.6 L_{B}}{L_{I}}\right)^{2}
$$

in good agreement with Vadzekis et al Finally, we compare the kinematical disk masses $M_{D}$ with those derived from the galaxy specto-photometry: $M_{\text {phot }}=$ $\mathcal{Y}_{I}(B-I) L_{I}$ (let us remind that $\mathcal{Y}_{I}$ is the average stellar mass-to-light ratio for a stellar population of color $B-I$. As a quite general outcome of the past history of galaxies, Vazdekis et al. 1996 found

$$
\mathcal{Y}_{I}=b(B-I)+c
$$

where $b \sim 0.8$ and the value of the constant $c$ is irrelevant. We find:

$$
M_{D}=(0.96 \pm 0.1) M_{\text {phot }}
$$

(28 d.o.f., see Fig 4), i.e. dynamical and photometric mass estimates statistically coincide. Notice that the disk mass and I-band luminosity are not directly proportional:

$$
\log M_{D}=(0.79 \pm 0.1) \log L_{I}+\text { const }
$$

with the log slope being significantly different from unity. Then, the existence of a relationship between the maximum rotation velocity and the galaxy luminosity is far from being a trivial one, in that the $I$-band luminosity is not a 


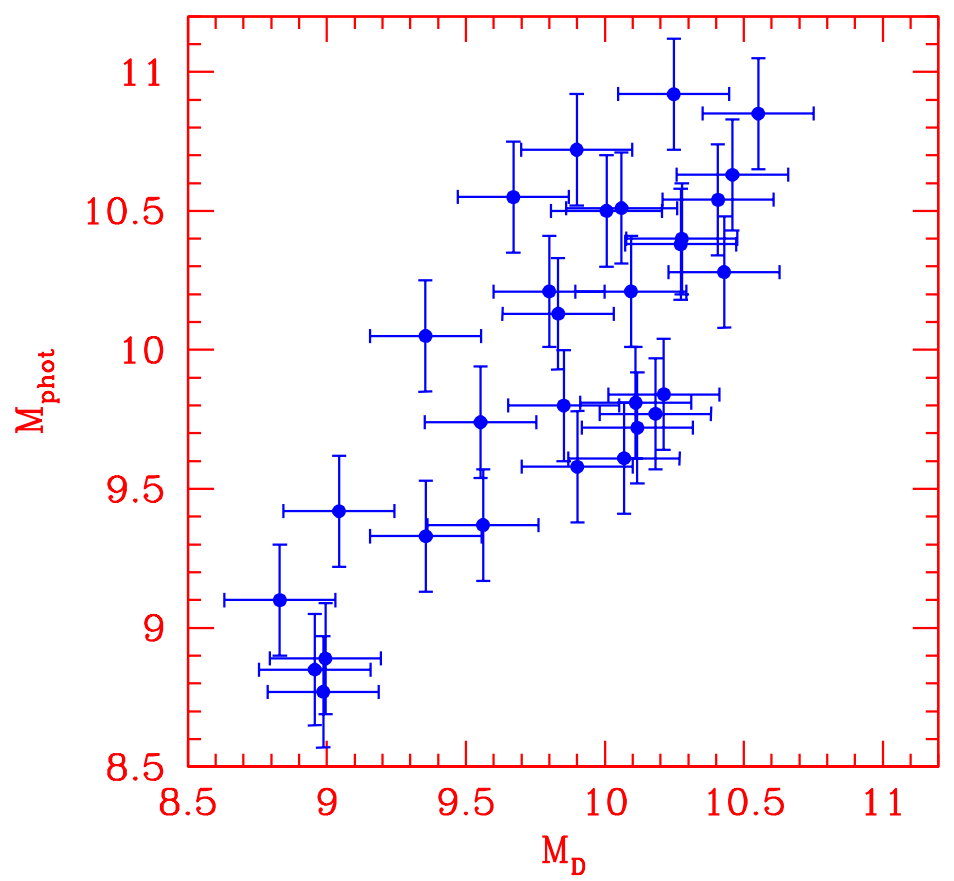

Fig. 4. Kinematical mass $M_{D}$ vs photometric disk masses. Units in $\log M_{\odot}$

straightforward measure of the stellar mass. It is worth to note that in the 3-D space defined by the $\left(\log L_{I}, \log \left(M_{D} / L_{I}\right), B-I\right)$ coordinate vector, spirals are not randomly distributed, but occupy a very thin plane (see Fig. 2).

\section{$4 \mathrm{MDO} / \mathrm{BH}$ masses. Upper limits}

The very good spatial resolution of the RC's of our sample plays a crucial role in the determination of strict and reliable MDO upper limits. In fact, since at small radii $V \propto R$ and the mass inside $r_{i n}$ scales as $r_{i n}^{3}$, a spatial resolution of $r_{i n} \sim 100 p c$ yields to a mass resolution of $\sim 10^{6} M_{\odot}$, given that $V\left(r_{i n}\right) \sim 10 \mathrm{~km} / \mathrm{s}$. In detail, the ratio of the rotational velocities $\left(V_{M D O} / V_{d}\right)^{2} \propto$ $\left(M_{M D O} / r_{i n}\right)^{3}$, implies that a higher spatial resolutions lead to stronger limits on $M_{M D O}$ (or easier detection).

We now force the presence of a central $\mathrm{MDO} / \mathrm{BH}$ by adopting the maximum possible MDO mass compatible with the rotation curve. We determine the upper mass limits by assuming $f=1$ or $f=f_{\max }$ with $f=f_{\max }$ the value that brings the model RC $1 \sigma$ higher than the innermost data. Note that for $f=1$ all the mass inside $r_{i n}$ is in the black hole. We typically find: $f_{\max }=0.7-0.8$ and that, in spite of having an additional parameter, the disk+black hole model performs significantly worse than the OD model. 


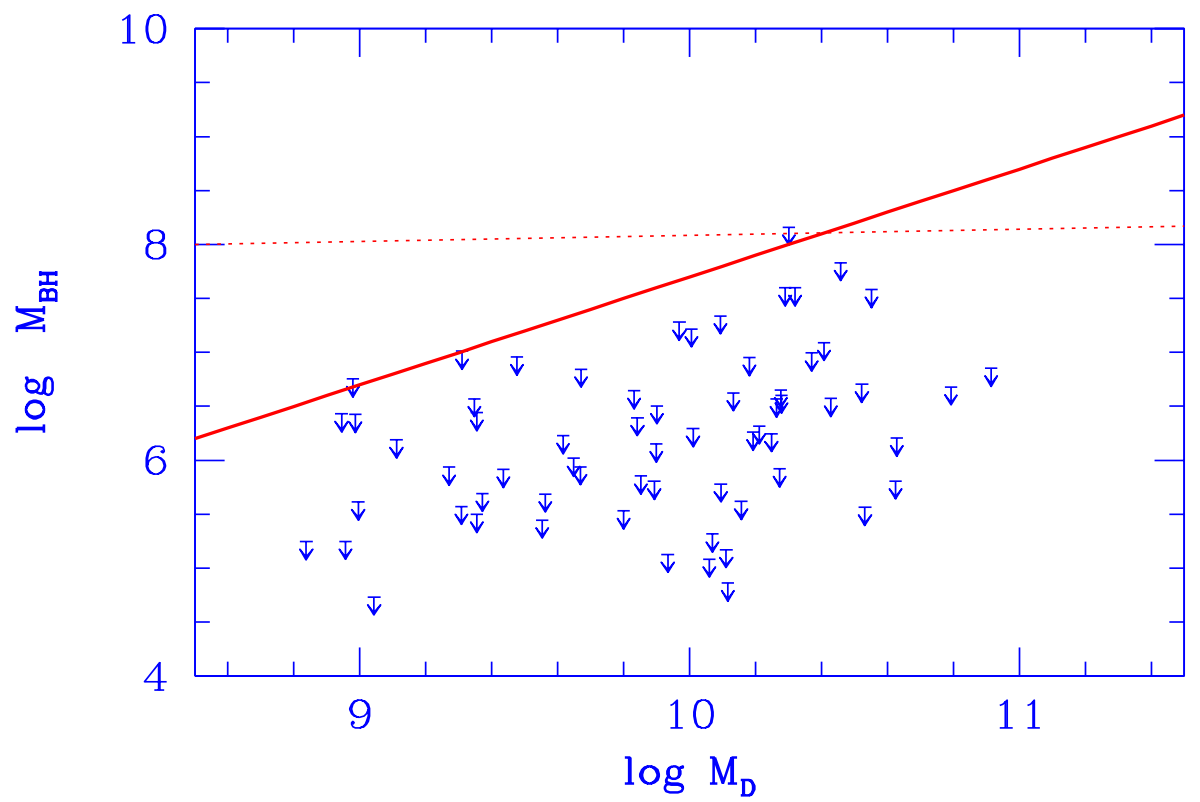

Fig. 5. Upper limits $\mathrm{BH} / \mathrm{MDO}$ masses vs. disk masses. The solid line is the $\mathrm{BH} / \mathrm{MDO}$ mass vs. stellar mass of ellipticals.

Notice that only in a few cases $f_{\max } \sim 0.2$, furthermore, no result changes if we assume $f_{\max }=1$ for all the objects. $M_{B H}$ is then obtained by substituting in equation $(2 \mathrm{~b})$ the corresponding values of $f$. These are shown in Table 1: it is evident that typically $M_{M D O}<<10^{8} M_{\odot}$ especially at low luminosity. It is clear that these limits are short of the mass residing in a accreting $\mathrm{BH}$ which is required to power high redshifts quasars, thus, spirals do not host QSO remnants (see also Salucci etal 2000). More specifically, if central MDO's of masses $\sim 2 M_{M D O}$ (and still $<10^{8} M_{\odot}$ ) were actually present at the center of the late type spirals of our sample, they would have affected the inner kpc of the available RC's more strongly than MDO's of $\sim 10^{9} M_{\odot}$ affect the inner kinematics of ellipticals (Magorrian et al., 1998).

In Fig. 5 we show the relationship $M_{B H}$ vs the stellar disk mass $M_{D}$, and, as a reference, we plot the extrapolation to spirals of the elliptical's stellar massMDO/BH mass relationship $M_{M D O}=0.005 \times M_{\text {stars }}$ (Magorrian et al.,1998, Kormendy \& Richstone 1995 ). We immediately realize, that in spirals, the MDO masses, if different from zero, are however much smaller than those detected at the centers of ellipticals of same stellar luminosity; whether this is related to their less prominent bulge content or does reflect also a morphological mass segregation, will be considered elsewhere (Salucci et al 2000). Notice that in the same paper we perform a detailed investigation on possible biases occurring in the present estimate of the MDO upper limits. 


\section{DISCUSSION}

Accurate mass models of the innermost kpc of 83 spirals derived from high spatial resolution RC's, reveal that, inside this region, the luminous matter fully accounts for the observed kinematics: a stellar disk of constant mass-tolight ratio (in some cases in conjunction with a spheroid) is virtually the only mass component. In this region, with the exception of very high luminosity galaxy with complex dynamics, we find

$$
V^{2}(R) \propto \operatorname{light}(<R) / R
$$

which is indicative of the absence of a sizable dark component. Therefore, the only possibility for the presence of a dark halo in the central regions of spirals is that it conceals itself below the detection threshold.

More in detail, inside the innermost kpc or so, the contribution to the gravitational potential from a dark component cannot exceed 10\%; this is in disagreement with claims and scenarios in which a dark halo has a major role at any radii. Moreover, in the same objects studied here, the DM, undetected at $R<1 / 3 R_{D}$, begins to dominate the mass distribution at $R \sim 3 R_{D}$ (PSS). Then, the dark mass inside a giv en radius $R$ must increase with $R$ very steeply, e.g. as in the case of a constant density distribution.

In a DM-free environment, we have been able to estimate the disk masses also for objects in which the global influence of the dark matter is relevant and to relate disk colors and masses across a large range of galaxy luminosities. The next step will be to pursue a coordinated kinematical and photometric study to tackle open cosmological issues.

\section{Appendix}

In the appendix we produce the two Tables of galaxy and MDO/BH properties. 


\section{References}

[1] Ghez A.M, Cline B.L, Morris M.,Becklin E.E., 1999,ApJ 509, 678

[2] Genzel R., AAS, 1998, 193, 620

[3] Ho L.C., 1998, in Observational Evidence for Black Holes in the Universe, ed. Chakrabarti, S. K., Kluwer Academic Pub.

[4] Kormendy J., Richstone D., 1995, ARA\&A, 33, 581

[5] Magorrian J., Tremaine S., Richstone D., Bender R., Bower G., Dressler A., Faber S.M., Gebhardt K., Green R., Grillmair C., Kormendy J., Lauer T.R., $1998,115,2285$

[6] Persic M., Salucci P., 1995 (PS95), ApJS, 99, 501

[7] Persic M., Salucci P., 1990, MNRAS, 245, 577

[8] Persic M., Salucci P., Stel F. (PSS), 1996, MNRAS, 281, 27

[9] Rhee M.H. 1997, Phd. Thesis, Groningen University

[10] Salucci P., Persic M., A\&A, 351, 442

[11] Salucci P., Ratnam C., Monaco P., Danese G., 2000 MNRAS in press

[12] Salucci P., Szuskiewicz E., Monaco P., Danese G., 1999 MNRAS, 307, 637

[13] Vazdekis A., Casuso E., Peletier R.F., Beckman J.E., 1996, ApJS, 106,307

[14] Vazdekis A., Peletier R.F., Beckman J.E., Casuso E., 1997, ApJS, 111,203 


\begin{tabular}{|c|c|c|c|c|}
\hline name & $M_{D}$ & B-I & $\mathcal{Y}$ & $L_{I}$ \\
\hline 116-G12 & 9.83 & .675 & .51 & 9.59 \\
\hline 121-G6 & 10.27 & .700 & .72 & 9.84 \\
\hline $143-\mathrm{G} 10$ & 8.83 & .225 & -.08 & 8.92 \\
\hline $346-\mathrm{G} 26$ & 10.11 & -.125 & .247 & 9.82 \\
\hline $347-G 28$ & 9.56 & -.150 & .01 & 9.49 \\
\hline 347-G33 & 10.40 & .300 & .23 & 10.30 \\
\hline 347-G34 & 10.27 & .025 & -.08 & 10.36 \\
\hline 357-G16 & 8.99 & -.350 & -.32 & 9.17 \\
\hline 359-G6 & 9.04 & -.100 & -.5 & 9.50 \\
\hline $362-\mathrm{G} 11$ & 9.89 & .775 & .11 & 10.10 \\
\hline 374-G8 & 8.95 & .000 & .11 & 8.85 \\
\hline $380-\mathrm{G} 23$ & 9.67 & .875 & .17 & 9.85 \\
\hline 406-G26 & 10.11 & .000 & .30 & 9.81 \\
\hline 418-G1 & 9.85 & .050 & .11 & 9.76 \\
\hline 441-G2 & 10.18 & -.050 & .35 & 9.81 \\
\hline 468-G23 & 9.55 & .000 & -.19 & 9.74 \\
\hline 487-G2 & 10.00 & .525 & .14 & 10.08 \\
\hline 488-G54 & 10.21 & -.250 & .07 & 10.04 \\
\hline 51-G18 & 10.06 & -.075 & .37 & 9.67 \\
\hline 533-G53 & 10.06 & .125 & -.30 & 10.41 \\
\hline 54-G21 & 9.35 & .375 & -.25 & 9.75 \\
\hline 554-G28 & 9.35 & .075 & .12 & 9.27 \\
\hline 556-G23 & 10.09 & .200 & .12 & 10.05 \\
\hline 566-G22 & 9.90 & -.150 & .14 & 9.70 \\
\hline $60-\mathrm{G} 25$ & 8.98 & -.250 & -.08 & 8.97 \\
\hline 84-G10 & 9.80 & .050 & -.35 & 10.17 \\
\hline 305-G6 & 10.45 & .250 & .13 & 10.43 \\
\hline 476-G15 & 10.42 & -.025 & .12 & 10.30 \\
\hline 79-G14 & 10.55 & .525 & .33 & 10.43 \\
\hline 79-G3 & 10.24 & .800 & .29 & 10.28 \\
\hline
\end{tabular}

Table 1

(1) Galaxy name (2) Logarithm of the disk mass in solar units (3) B-I color (4) Logarithm of the disk mass-to-light ratio (I-band). (5) I-Band luminosity. 


\begin{tabular}{|c|c|c|c|c|c|}
\hline name & $\log M_{B H}$ & name & $\log M_{B H}$ & name & $\log M_{B H}$ \\
\hline 116-g12 & 6.6 & 418-g8 & 6.4 & $\mathrm{~m}-1-2524$ & 7.0 \\
\hline 121-g6 & 6.7 & 436-g3 & 6.7 & m-214003 & 6.3 \\
\hline 13-g16 & 5.9 & 441-g2 & 7.0 & m-3-1042 & 5.8 \\
\hline 143-g10 & 5.2 & $446-g 53$ & 6.8 & n1090 & 5.6 \\
\hline 157-g20 & 7.2 & 468-g23 & 5.4 & n1832 & 8.3 \\
\hline 157-g38 & 5.9 & 481-g13 & 5.6 & n2763 & 7.6 \\
\hline 162-g17 & 6.2 & 487-g2 & 7.2 & n3715 & 8.2 \\
\hline $233-g 42$ & 6.3 & 488-g54 & 6.3 & n4348 & 6.2 \\
\hline 249-g16 & 5.8 & 490-g28 & 5.9 & n699 & 6.3 \\
\hline 249-g35 & 5.5 & $490-\mathrm{g} 45$ & 7.3 & n7218 & 7.6 \\
\hline 265-g2 & 6.7 & 498-g3 & 6.6 & n7339 & 6.6 \\
\hline 302-g9 & 6.6 & 51-g18 & 5.3 & n755 & 5.7 \\
\hline $328-g 43$ & 6.2 & 533-g53 & 5.1 & ua17 & 5.8 \\
\hline 329-g7 & 7.2 & 54-g21 & 5.5 & 269-g19 & 8.3 \\
\hline $346-g 26$ & 4.9 & 545-g3 & 5.1 & $305-\mathrm{g} 6$ & 7.8 \\
\hline 347-g28 & 5.7 & 545-g5 & 6.4 & $358-g 63$ & 6.7 \\
\hline $347-g 33$ & 7.1 & 547-g24 & 7.0 & 36-g19 & 7.7 \\
\hline 347-g34 & 5.9 & $55-\mathrm{g} 4$ & 6.3 & 476-g15 & 6.6 \\
\hline $35-g 18$ & 7.7 & $550-\mathrm{g} 7$ & 5.6 & 545-g11 & 8.3 \\
\hline $357-g 16$ & 5.6 & 554-g28 & 6.4 & 547-g31 & 7.4 \\
\hline $359-\mathrm{g} 6$ & 4.7 & 556-g23 & 7.3 & 582-g12 & 7.7 \\
\hline $362-g 11$ & 6.2 & 563-g14 & 7.3 & 79-g14 & 7.6 \\
\hline 374-g8 & 5.2 & 566-g22 & 6.5 & 79-g3 & 6.2 \\
\hline $380-g 23$ & 6.8 & 575 -g53 & 6.0 & i96099 & 6.9 \\
\hline 406-g26 & 5.2 & 58-g30 & 6.6 & $\mathrm{~m}-222025$ & 8.6 \\
\hline 410-g19 & 7.0 & 60-g25 & 6.4 & n4705 & 7.2 \\
\hline 418-g1 & 5.9 & 84-g10 & 5.5 & n697 & 8.4 \\
\hline I407 & 7. & I96099 & 6.9 & & \\
\hline
\end{tabular}

Table 2

$\mathrm{BH} / \mathrm{MDO}$ upper limits for the galaxies of the sample, in units of $\log M_{\odot}$ 